\title{
Effect of Orally Administered Semaglutide Versus Dulaglutide on Diabetes-Related Quality of Life in Japanese Patients with Type 2 Diabetes: The PIONEER 10 Randomized, Active-Controlled Trial
}

\author{
Hitoshi Ishii (D) · Brian B. Hansen · Jakob Langer · Hiroshi Horio
}

Received: November 16, 2020 / Accepted: December 11, 2020 / Published online: January 18, 2021

(C) The Author(s) 2021

\begin{abstract}
Introduction: In the randomized Peptide InnOvatioN for Early diabEtes tReatment (PIONEER) 10 trial, once-daily orally administered semaglutide-the first oral glucagon-like peptide 1 receptor agonist (GLP-1RA)-was similarly tolerated with comparable (at $7 \mathrm{mg}$ ) or better (at $14 \mathrm{mg}$ ) efficacy versus the injectable GLP-1RA dulaglutide $0.75 \mathrm{mg}$. Health-related quality of life (HRQoL) in PIONEER 10 was assessed using the Japanesespecific Diabetes Therapy-Related Quality of Life (DTR-QoL) questionnaire.

Methods: The DTR-QoL comprises 29 questions, providing four domain and total scores. Answers were converted to a score between 0 and 100, with higher scores indicating greater HRQoL. Two estimands were prespecified: treatment policy (regardless of treatment
\end{abstract}

Supplementary Information The online version contains supplementary material available at https:// doi.org/10.1007/s13300-020-00985-w.

H. Ishii $(\bowtie)$

Department of Doctor-Patient Relationships, Nara

Medical University, Nara, Japan

e-mail: hits1@naramed-u.ac.jp

B. B. Hansen

Novo Nordisk A/S, Søborg, Denmark

J. Langer · H. Horio

Novo Nordisk Pharma Ltd, Tokyo, Japan discontinuation or rescue medication use) and trial product (assuming on treatment without rescue medication) in all randomized patients. Outcomes were assessed at weeks 26 and 52.

Results: Mean baseline DTR-QoL domain scores were similar between treatment arms and were generally lower (giving more scope for improvement) for "anxiety and dissatisfaction with treatment" (62.1-65.3) and "satisfaction with treatment" (53.9-57.9) than "burden on social activities and daily activities" (76.5-77.7) and "hypoglycemia" (83.5-88.2). Using the treatment policy estimand, orally administered semaglutide 7 and $14 \mathrm{mg}$ improved HRQoL versus dulaglutide $0.75 \mathrm{mg}$ for the total score (estimated mean change from baseline [CfB] 7.3 and 8.1 vs 3.3; estimated treatment difference [ETD] 3.9 and 4.8) and the "anxiety and dissatisfaction with treatment" domain (CfB 9.7 and 10.9 vs 3.7; ETD 6.0 and 7.2) at week 52 . Orally administered semaglutide $14 \mathrm{mg}$ improved the "satisfaction with treatment" domain versus dulaglutide $0.75 \mathrm{mg}$ (CFB 13.8 vs 5.7; ETD 8.1). DTR-QoL scores for orally administered semaglutide tended to be more durable (sustained over time) than for dulaglutide. Outcomes for the trial product estimand were similar.

Conclusion: Orally administered semaglutide 7 and $14 \mathrm{mg}$ improved the patients' HRQoL measured by the Japanese-specific DTR-QoL instrument versus dulaglutide $0.75 \mathrm{mg}$ at week 52. 
Trial

NCT03015220.

registration: ClinicalTrials.gov

Keywords: Clinical trial; Diabetes TreatmentRelated Quality of Life; Dulaglutide; GLP-1 receptor agonist; Health-related quality of life; Japan; Orally administered semaglutide; Phase 3; Type 2 diabetes

\section{Key Summary Points}

Why carry out this study?

Orally administered semaglutide is the first oral glucagon-like peptide 1 receptor agonist (GLP-1RA) and has been developed to provide an option for people with type 2 diabetes (T2D) who could benefit from this class of treatment but who may not wish to initiate injection therapy

Orally administered semaglutide has been shown to be effective and well tolerated compared with other oral and injectable glucose-lowering medications in phase 3 trials; the comparative effect on health-related quality of life is also important

\section{What did the study ask?}

The randomized PIONEER 10 trial assessed the efficacy and safety of once-daily orally administered semaglutide compared with the once-weekly GLP-1RA dulaglutide in Japanese patients with T2D; here, we report health-related quality of life outcomes from this trial assessed using the Japanese-specific Diabetes TherapyRelated Quality of Life (DTR-QoL) questionnaire

\section{What was learned from the study?}

Orally administered semaglutide 7 and $14 \mathrm{mg}$ improved the total DTR-QoL score to a greater extent than dulaglutide $0.75 \mathrm{mg}$ after 52 weeks' treatment, as well as the individual domain of "anxiety and dissatisfaction with treatment"; furthermore, orally administered semaglutide $14 \mathrm{mg}$ improved the "satisfaction with treatment" domain versus dulaglutide $0.75 \mathrm{mg}$

DTR-QoL scores for orally administered semaglutide tended to be more durable (sustained over time), with similar scores at weeks 26 and 52, whereas scores declined for dulaglutide between weeks 26 and 52

\section{DIGITAL FEATURES}

This article is published with digital features, including a summary slide, to facilitate understanding of the article. To view digital features for this article go to https://doi.org/10.6084/ m9.figshare.13360163.

\section{INTRODUCTION}

Semaglutide is one of a number of glucagon-like peptide 1 receptor agonists (GLP-1RAs) recommended for the control of hyperglycemia in patients with type 2 diabetes (T2D) [1, 2]. Of these agents, subcutaneously administered once-weekly semaglutide, once-weekly dulaglutide, and once-daily liraglutide are also indicated for patients with T2D and established cardiovascular disease [3-5], as they have been shown to reduce the risk of major adverse cardiovascular events in this population [6-8].

Semaglutide is the first and, so far, only GLP1RA to be formulated in a tablet, and uses the absorption enhancer sodium $\mathrm{N}$-(8-[2-hydroxybenzoyl]amino)caprylate (SNAC) to protect semaglutide from enzymatic degradation and 
facilitate its gastric absorption after oral administration [9]. Prior to the development of orally administered semaglutide, all GLP-1RAs were administered by injection. On the basis of experiences with insulin, the injectable route of administration may discourage some patients and healthcare providers from using these agents when oral glucose-lowering therapies are available [10, 11]. Among the injectable GLP1RAs, those that are administered once weekly were associated with improved health-related quality of life (HRQoL) compared with those given more frequently [12]. Orally administered semaglutide may help more patients with uncontrolled T2D to access GLP-1RA therapy [13] by overcoming some of the barriers associated with injectable therapies, and has now been approved for once-daily dosing in Japan [14] and elsewhere [15, 16].

Orally administered semaglutide was effective and well tolerated in the phase 3 a PIONEER clinical trial program, which comprised 10 studies [17-26], including two dedicated trials in Japanese patients $[25,26]$. One of the two Japanese trials, PIONEER 10, compared oncedaily orally administered semaglutide with once-weekly dulaglutide $0.75 \mathrm{mg}$ in patients whose T2D was inadequately controlled on one existing oral glucose-lowering drug [26]. In this trial, orally administered semaglutide $7 \mathrm{mg}$ had similar glycemic efficacy, but greater body weight reductions, and orally administered semaglutide $14 \mathrm{mg}$ had significantly greater reductions in glycated hemoglobin $\left(\mathrm{HbA}_{1 \mathrm{c}}\right)$ and body weight, than dulaglutide $0.75 \mathrm{mg}$ after 26 and 52 weeks' treatment [26].

Several globally used measures of HRQoL were employed in the PIONEER program. These scales are potentially useful but do not take account of potential differences between Japanese and global patients with T2D [27]. In PIONEER 10, the effect of treatment on patients' HRQoL was assessed using the validated and widely used Japan-specific Diabetes Therapy-Related Quality of Life (DTR-QoL) questionnaire [28]. Here, we present the full results of these Japanese disease-specific patientreported outcomes.

\section{METHODS}

\section{PIONEER 10 Study Design}

The full methods and primary results of the PIONEER 10 trial are reported in the primary manuscript [26]. In brief, this was a 52-week randomized, open-label, active-controlled, parallel-group, multicenter, phase 3a trial carried out in Japan. Japanese adults aged at least 20 years with $\mathrm{T} 2 \mathrm{D}$, and with $\mathrm{HbA}_{1 \mathrm{c}} 7.0-10.5 \%$ (53-91 $\mathrm{mmol} / \mathrm{mol}$ ) despite receiving stable oral glucose-lowering monotherapy (sulfonylurea, glinide, thiazolidinedione, $\alpha$-glucosidase inhibitor, or sodium-glucose co-transporter-2 inhibitor [SGLT2i]) were eligible to participate. Patients were randomized 2:2:2:1 to receive either once-daily orally administered semaglutide 3,7 , or $14 \mathrm{mg}$, or once-weekly subcutaneously administered dulaglutide $0.75 \mathrm{mg}$, as add-on to their background medication; efficacy was assessed at 26 weeks and at the end of treatment at 52 weeks.

Institutional review boards/independent ethics committees at each site provided approval before the start of the trial and all patients provided informed consent. The trial was conducted according to relevant national requirements, and complied with the Declaration of Helsinki and International Conference on Harmonisation Guidelines for Good Clinical Practice. Full information on study ethics is reported in the primary manuscript [26].

\section{Assessment of Diabetes Therapy-Related Quality of Life Scale}

The DTR-QoL questionnaire is a Japanese diabetes-specific HRQoL measure that contains 29 items (Table S1) to assess the influence of diabetes treatment on HRQoL across four domains [28]: burden on social activities and daily activities; anxiety and dissatisfaction with treatment; hypoglycemia; and satisfaction with treatment.

The questionnaire was delivered in Japanese and was ideally answered before any other trialrelated activities. Patients were able to complete 
the questionnaire by themselves without interruption.

The questionnaire was completed at baseline, and after 26 and 52 weeks. Scores were converted to a 1-100 scale, with higher numbers indicating greater diabetes-related quality of life. A total score was derived on the basis of the responses to all questions. This manuscript will focus on the results after 52 weeks of treatment to align with the reported clinical outcomes, but data after 26 weeks are also presented.

The approved maintenance dose for orally administered semaglutide in Japan is $7 \mathrm{mg}$ once daily, with the option of increasing this to $14 \mathrm{mg}$ if glycemic control remains insufficient [14]; in Europe and the USA, either 7 or $14 \mathrm{mg}$ may be used depending on glycemic efficacy $[15,16]$. Therefore, we focus on the DTR-QoL outcomes for the 7 and $14 \mathrm{mg}$ doses below, but results for the $3 \mathrm{mg}$ dose are also presented.

In the PIONEER trials, two scientific questions related to the efficacy objectives (including patient-reported outcomes) were addressed through the definition of two estimands ("treatment policy" and "trial product") [29]. The treatment policy estimand evaluated the treatment effect for all randomized patients, regardless of trial product discontinuation or use of rescue medication and was the primary estimand in PIONEER 10. The trial product estimand evaluated the treatment effect for all randomized patients, under the assumption that all patients remained on trial product for the entire planned duration of the trial and did not use rescue medication. Here, DTR-QoL results for the treatment policy estimand are the focus.

\section{Statistical Analysis}

As previously explained [26], patient-reported outcomes were analyzed as follows, with stratification factor (background oral glucose-lowering medication at screening) as a categorical fixed effect and baseline value as a covariate. The treatment policy estimand was estimated by a pattern mixture model using multiple imputation to handle missing data at weeks 26 and 52. Data were collected from all randomized patients, irrespective of premature discontinuation of trial product or initiation of rescue medication. Imputation was done within five groups: one group of patients who had discontinued treatment or initiated rescue medication at the analysis time point (either week 26 or 52), regardless of randomized treatment arm; and four groups of patients who were still taking trial product without rescue medication, defined by randomized treatment arm. Both the imputation and the analysis were based on analysis of covariance models. The results were combined by use of Rubin's rule [30]. The trial product estimand was estimated by a mixed model for repeated measurements that used data collected prior to premature trial product discontinuation or initiation of rescue medication from all randomized patients.

Results are presented as estimated change from baseline in DTR-QoL scores and estimated treatment differences (ETDs) with 95\% confidence intervals (CIs). Statistical analyses were not controlled for multiplicity. All analyses were performed using SAS version 9.4M2.

\section{RESULTS}

\section{Participants}

As previously reported, 458 patients were randomized to orally administered semaglutide $3 \mathrm{mg} \quad(n=131), \quad 7 \mathrm{mg} \quad(n=132), \quad$ or $14 \mathrm{mg}$ $(n=130)$, or dulaglutide $0.75 \mathrm{mg}(n=65)$ [26]. Baseline clinical characteristics are summarized in Table S2 in the supplementary appendix. Baseline mean DTR-QoL domain scores were similar between the treatment arms (Table 1), but were generally lower for "anxiety and dissatisfaction from treatment" (62.1-65.3) and "satisfaction with treatment" (53.9-57.9), compared with "burden on social activities and daily activities" (76.5-77.7), and "hypoglycemia" (83.5-88.2). 
Table 1 Baseline DTR-QoL domain scores

\begin{tabular}{|c|c|c|c|c|}
\hline & $\begin{array}{l}\text { Orally administered } \\
\text { semaglutide } 3 \mathrm{mg}\end{array}$ & $\begin{array}{l}\text { Orally administered } \\
\text { semaglutide } 7 \mathrm{mg}\end{array}$ & $\begin{array}{l}\text { Orally administered } \\
\text { semaglutide } 14 \mathrm{mg}\end{array}$ & $\begin{array}{l}\text { Dulaglutide } \\
0.75 \mathrm{mg}\end{array}$ \\
\hline Number of patients & 131 & 132 & 130 & 65 \\
\hline $\begin{array}{l}\text { Burden on social activities } \\
\text { and daily activities }\end{array}$ & $76.9 \pm 19.0$ & $76.5 \pm 18.4$ & $77.7 \pm 16.2$ & $77.1 \pm 19.1$ \\
\hline $\begin{array}{l}\text { Anxiety and dissatisfaction } \\
\text { with treatment }\end{array}$ & $64.6 \pm 21.5$ & $62.7 \pm 20.9$ & $62.1 \pm 20.0$ & $65.3 \pm 23.6$ \\
\hline Hypoglycemia & $83.5 \pm 20.0$ & $88.2 \pm 16.6$ & $85.3 \pm 20.6$ & $86.3 \pm 18.2$ \\
\hline Satisfaction with treatment & $55.6 \pm 18.3$ & $57.9 \pm 17.2$ & $53.9 \pm 20.7$ & $54.2 \pm 20.9$ \\
\hline Total score & $71.5 \pm 15.7$ & $71.7 \pm 14.8$ & $71.2 \pm 14.1$ & $71.9 \pm 16.2$ \\
\hline
\end{tabular}

Values are mean \pm standard deviation unless otherwise stated

DTR-QoL Diabetes Therapy-Related Quality of Life

\section{DTR-QoL Outcomes}

Outcomes for the four domains and total score are shown in Fig. 1 for the treatment policy (primary) estimand. Data were imputed for less than $5 \%$ of patients. Observed changes from baseline in the scores for each question are shown in Table $\mathrm{S} 1$, and ETDs in the changes from baseline in the four domains and total score are shown in Table S3.

\section{Burden on Social Activities and Daily Activities} Improvements in burden on social activities and daily activities were not significantly different (lower bound of 95\% CI for the ETD crossed zero) with orally administered semaglutide 7 and $14 \mathrm{mg}$ compared with dulaglutide $0.75 \mathrm{mg}$ at week 26 (change from baseline 6.5 and 5.1 points, respectively, vs 4.6 points) and week 52 (6.6 and 6.1 vs 3.6) for the treatment policy estimand (Fig. 1i). The higher numerical values for orally administered semaglutide appear to have been influenced by the scoring of questions concerning managing treatment and associated pain/discomfort of injections, such as "my current diabetes treatment interferes with my work and activities" and "pain due to my current diabetes treatment is uncomfortable" (Table S1). There seemed to be no negative impact of the dosing and administration regimen of once-daily orally administered semaglutide on the perception of burden compared with once-weekly subcutaneously administered dulaglutide $0.75 \mathrm{mg}$.

\section{Anxiety and Dissatisfaction with Treatment}

The changes from baseline in scores for anxiety and dissatisfaction with treatment for orally administered semaglutide 7 and $14 \mathrm{mg}$ compared with dulaglutide $0.75 \mathrm{mg}$ (10.5 and 11.6, respectively, vs 9.2) were not significantly different at week 26 (Fig. 1ii). At week 52, the scores (9.7 and 10.9 vs 3.7 ) indicated that orally administered semaglutide 7 and $14 \mathrm{mg}$ were associated with significantly reduced anxiety and dissatisfaction with treatment versus dulaglutide $0.75 \mathrm{mg}$ (ETD 6.0; 95\% CI 0.8, 11.3; and ETD 7.2; 95\% CI 1.9, 12.5; respectively) (Table S3). In particular, concerns about weight gain and control of blood glucose appear to have driven the difference between outcomes for orally administered semaglutide 7 and $14 \mathrm{mg}$ compared with dulaglutide $0.75 \mathrm{mg}$ (Table S1).

\section{Hypoglycemia}

Scores for patient perceptions of hypoglycemia were similar between orally administered semaglutide 7 and $14 \mathrm{mg}$ compared with dulaglutide $0.75 \mathrm{mg}$ at week 26 (change in 

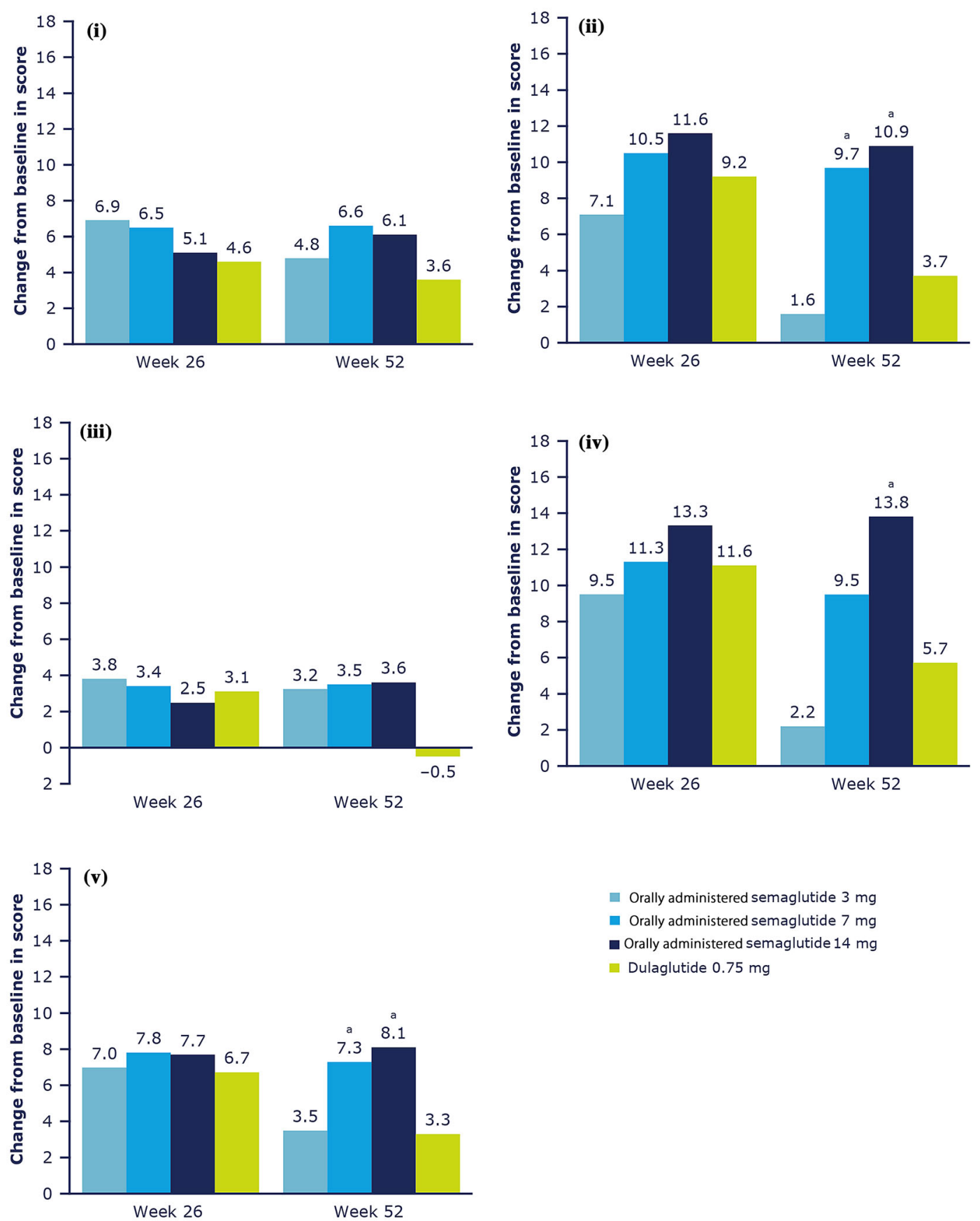
- Orally administered semaglutide $3 \mathrm{mg}$
- Orally administered semaglutide $7 \mathrm{mg}$
- Orally administered semaglutide $14 \mathrm{mg}$
- Dulaglutide $0.75 \mathrm{mg}$


4Fig. 1 Estimated changes from baseline in DTR-QoL domain scores at weeks 26 and 52 for the treatment policy estimand. (i) Burden on social activities and daily activities; (ii) anxiety and dissatisfaction with treatment; (iii) hypoglycemia; (iv) satisfaction with treatment; and (v) total score. Data are for the treatment policy estimand. Missing post-baseline values were imputed by a pattern mixture model using multiple imputation. Pattern was defined by treatment arm and treatment status (premature trial product discontinuation and/or initiation of rescue medication), and imputations were based on an ANCOVA model. Imputation was from own treatment arm and same treatment status. Change from baseline was analyzed using an ANCOVA model with treatment and strata as categorical fixed effects and baseline value as the covariate for each of the 1000 imputed complete datasets, and pooled by Rubin's rule to draw inference [30]. No statistical analyses were controlled for multiplicity as there

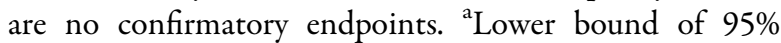
confidence interval $>0$ for the estimated treatment difference vs dulaglutide $0.75 \mathrm{mg}$, favoring orally administered semaglutide. $A N C O V A$ analysis of covariance, DTR-QoL Diabetes Therapy-Related Quality of Life

scores from baseline 3.4 and 2.5, respectively, vs 3.1) and were not statistically different at week 52 (3.5 and 3.6 vs -0.5 ) (Fig. 1iii and Table S1). However, whereas the improvement in hypoglycemia score from baseline with all orally administered semaglutide doses remained similar between week 26 and week 52, the score for dulaglutide $0.75 \mathrm{mg}$ decreased (worsened).

\section{Satisfaction with Treatment}

At week 26, the change from baseline in score for satisfaction with treatment was similar between orally administered semaglutide 7 and $14 \mathrm{mg}$ (11.3 and 13.3) and dulaglutide $0.75 \mathrm{mg}$ (11.6) (Fig. 1iv). At week 52, orally administered semaglutide $14 \mathrm{mg}$ (change from baseline in score 13.8) was associated with significantly greater satisfaction with treatment compared with dulaglutide $0.75 \mathrm{mg}$ (5.7) at week 52 (ETD 8.1; 95\% CI 1.9, 14.3) (Table S3). Patients receiving orally administered semaglutide $14 \mathrm{mg}$ recorded higher improvements in questions regarding blood glucose control, satisfaction, and hope for the future compared with dulaglutide $0.75 \mathrm{mg}$ (Table S1).

\section{Total Score}

At week 26, the overall change from baseline in DTR-QoL score was similar between orally administered semaglutide 7 and $14 \mathrm{mg}$ compared with dulaglutide $0.75 \mathrm{mg}$ (estimated change from baseline 7.8 and 7.7, respectively, vs 6.7) (Fig. 1v). At week 52, the score for dulaglutide $0.75 \mathrm{mg}$ (3.3) was decreased versus week 26 , whereas scores for orally administered semaglutide $7 \mathrm{mg} \quad$ (7.3) and $14 \mathrm{mg}$ (8.1) remained durable between week 26 and 52 . Orally administered semaglutide $7 \mathrm{mg}$ and $14 \mathrm{mg}$ were associated with significantly increased total DTR-QoL scores at week 52 from baseline compared with dulaglutide $0.75 \mathrm{mg}$ (ETD 3.9; 95\% CI 0.2, 7.7; and ETD 4.8; 95\% CI 1.0, 8.6; respectively) (Table $\mathrm{S} 3$ ).

DTR-QoL scores and estimated treatment differences between orally administered semaglutide and dulaglutide for the individual domains and the overall score were generally similar to those reported above for the treatment policy estimand when assessed using the trial product estimand (Fig. S1). For the trial product estimand, only orally administered semaglutide $14 \mathrm{mg}$ significantly increased total score versus dulaglutide $0.75 \mathrm{mg}$ at week 52 .

\section{DISCUSSION}

Orally administered semaglutide 7 and $14 \mathrm{mg}$ showed greater improvements in Japanese patients' DTR-QoL scores at week 52 compared with dulaglutide $0.75 \mathrm{mg}$. DTR-QoL scores for orally administered semaglutide tended to be more durable (sustained over time), with similar scores at weeks 26 and 52, whereas scores declined for dulaglutide $0.75 \mathrm{mg}$ between weeks 26 and 52 across the four domains and for the total score. It should be noted that the greatest room for improvement was in the domains of "satisfaction with treatment" and "anxiety and dissatisfaction with treatment", whereas the high baseline scores in the other domains may have contributed to a limited scope for improvement with treatment.

Both western and Japanese patients are known to have concerns about injectable therapy with insulin $[10,11]$, and Japanese patients 
have been shown to have a higher preference for an oral, rather than injectable, GLP-1RA drug profile [31]. Orally administered semaglutide has been formulated to address this barrier, but has specific dosing requirements because of the effect of food, liquid, and concomitant medication on its absorption [9]. In PIONEER 10, patients were instructed to take their tablet in the morning, fasted, with no more than approximately $120 \mathrm{~mL}$ of water, and then to wait at least $30 \mathrm{~min}$ before consuming any food, other beverage, or other oral medication [26]. The change from baseline scores for the domain "burden on social activities and daily activities" were numerically (but not statistically) higher (better) with orally administered semaglutide 7 and $14 \mathrm{mg}$ compared with dulaglutide $0.75 \mathrm{mg}$, indicating that patients considered the once-daily orally administered semaglutide schedule to be, at least, no more burdensome than a once-weekly injection of dulaglutide. This clinically important observation is supported by previous work suggesting that the treatment burden associated with oncedaily oral therapy may be similar to that for once-weekly injections [32]. Individual question scores for this domain indicated concerns around managing injectable treatment and any associated pain or discomfort. The preference for oral therapy among Japanese patients [31] could also have contributed to the durable effect of orally administered semaglutide on HRQoL over 52 weeks, compared with the decline in score observed with dulaglutide. Of note, in the global PIONEER 7 trial, patients answering the Diabetes Treatment Satisfaction Questionnaire reported similar treatment satisfaction with orally administered semaglutide and orally administered sitagliptin after 52 weeks, regardless of the dosing conditions for orally administered semaglutide [23].

Domain scores closely reflected the objective primary clinical outcomes $\left(\mathrm{HbA}_{1 \mathrm{c}}\right.$ and body weight reduction) in the PIONEER 10 trial [26], suggesting a high sensitivity for the DTR-QoL questionnaire in measuring diabetes-related HRQoL. The domain "anxiety and dissatisfaction with treatment" included the statements "I am bothered by weight gain with my current diabetes treatment", "I am worried about high blood sugar", and "I am dissatisfied that my blood sugar is unstable (high and low)"; higher scores for orally administered semaglutide 7 and $14 \mathrm{mg}$ versus dulaglutide $0.75 \mathrm{mg}$ in scores for this domain (reaching statistical significance at week 52) appeared to align with the significantly greater reductions in $\mathrm{HbA}_{1 \mathrm{c}}$ seen with orally administered semaglutide $14 \mathrm{mg}$, and body weight with both the 7 and $14 \mathrm{mg}$ doses, versus dulaglutide $0.75 \mathrm{mg}$ at weeks 26 and 52 . Patients gained, on average, $1 \mathrm{~kg}$ of body weight with dulaglutide $0.75 \mathrm{mg}$ at week 52 compared with baseline, whereas they lost weight with orally administered semaglutide $7 \mathrm{mg}(-0.9 \mathrm{~kg})$ and $14 \mathrm{mg}(-1.6 \mathrm{~kg})$, assessed using the treatment policy estimand [26]. These effects on the clinical outcomes could have contributed to the decline in HRQoL between weeks 26 and 52 with dulaglutide, measured by the DTR-QoL.

The low and similar changes from baseline across treatments for the domain "hypoglycemia" correlated with the fact that there were few severe or hypoglycemic events confirmed by blood glucose during orally administered semaglutide or dulaglutide treatment [26]; in addition, baseline scores were high, indicating a higher quality of life for this domain at the start of the trial.

Change from baseline scores for the "satisfaction with treatment" domain remained largely stable for orally administered semaglutide 7 and $14 \mathrm{mg}$, but worsened with dulaglutide $0.75 \mathrm{mg}$ between weeks 26 and 52. This domain included the statements "Overall, I am satisfied with my current blood sugar control" and "I am confident that I can maintain good blood sugar control with my current diabetes treatment". The difference between orally administered semaglutide $14 \mathrm{mg}$ and dulaglutide $0.75 \mathrm{mg}$ at week 52 for this domain reflected a statistically significantly greater reduction in $\mathrm{HbA}_{1 \mathrm{c}}$ between treatments [26]. Patients' scores for the statements "I am hopeful about the future with my current diabetes treatment" and "I am satisfied with my current treatment methods as diabetes treatment" also contributed to overall treatment satisfaction with orally administered semaglutide.

The DTR-QoL is a Japanese-specific measure of HRQoL that has been extensively employed 
in studies of glucose-lowering medication, including several involving GLP-1RAs [33-35], dipeptidyl peptidase-4 inhibitors [36-38], and the SGLT2i ipragliflozin [39]. As in the current trial, the questionnaire has shown a high sensitivity for detecting and measuring diabetesrelated HRQoL compared with, for example, the EQ-5D questionnaire [40]. In PIONEER 9, similar DTR-QoL domain and total scores were recorded for orally administered semaglutide 3 , 7 , and $14 \mathrm{mg}$ compared with subcutaneously administered liraglutide at the Japanese-approved dose of $0.9 \mathrm{mg}$ once daily, when both were given as monotherapy [25].

The background medications allowed in this trial were reflective of a typical first-line pharmacotherapeutic approach to glucose-lowering treatment in Japanese patients with type 2 diabetes. Data were imputed for less than $5 \%$ of patients, which was unlikely to substantially affect the results. Nevertheless, a limitation of the current study is that the potential difference in adherence between a clinical trial and the real-world setting may lead to differences in HRQOL and treatment satisfaction for orally administered semaglutide, liraglutide, and dulaglutide. A further limitation is that the statistical analyses were not controlled for multiplicity.

\section{CONCLUSION}

Among Japanese patients with T2D receiving one background oral antidiabetic drug, treatment with once-daily orally administered semaglutide 7 and $14 \mathrm{mg}$ was associated with improved HRQoL compared with once-weekly subcutaneously administered dulaglutide $0.75 \mathrm{mg}$. The DTR-QoL has a high degree of sensitivity for measuring diabetes-related HRQoL, as demonstrated by the close alignment between the domain scores and the objective clinical outcomes. Orally administered semaglutide may help more patients with T2D to benefit from GLP-1RA therapy.

\section{ACKNOWLEDGEMENTS}

We gratefully thank the patients taking part in this trial, the investigators, all trial site staff, and all Novo Nordisk employees involved in the trial.

Funding. Sponsorship for this study and fees for publication of this manuscript were funded by Novo Nordisk Pharma Ltd., Japan.

Medical Writing and/or Editorial Assistance. We would like to thank Stephen Purver of Axis, a division of Spirit Medical Communications Group Ltd for medical writing and editorial assistance (funded by Novo Nordisk A/S), and Srikanth Deenadayalan and Kazushiro Fujiwara of Novo Nordisk for reviewing the manuscript.

Authorship. All named authors meet the International Committee of Medical Journal Editors (ICMJE) criteria for authorship for this article, take responsibility for the integrity of the work as a whole, and have given their approval for this version to be published.

Prior Presentation. This study was presented at the Japanese Diabetes Society annual congress, October 5-16, 2020.

Disclosures. Hitoshi Ishii has received lecture fees from Novo Nordisk, Eli Lilly, Sanofi, Daiichi-Sankyo, Dainihon-Sumitmo, Takeda, Tanabe-Mitsubishi, Boehringer Ingelheim, MSD, and Ono. Jakob Langer and Hiroshi Horio are employees of Novo Nordisk Pharma Ltd., Japan. Brian Bekker Hansen is an employee and shareholder of Novo Nordisk A/S, Denmark.

Compliance with Ethics Guidelines. Institutional review boards/independent ethics committees at each site provided approval before the start of the trial and all patients provided informed consent. The trial was conducted according to relevant national requirements, and complied with the Declaration of Helsinki and International Conference on Harmonisation Guidelines for Good Clinical Practice. 
Data Availability. The datasets generated during and/or analyzed during the current study are available from the corresponding author on reasonable request.

Open Access. This article is licensed under a Creative Commons Attribution-NonCommercial 4.0 International License, which permits any non-commercial use, sharing, adaptation, distribution and reproduction in any medium or format, as long as you give appropriate credit to the original author(s) and the source, provide a link to the Creative Commons licence, and indicate if changes were made. The images or other third party material in this article are included in the article's Creative Commons licence, unless indicated otherwise in a credit line to the material. If material is not included in the article's Creative Commons licence and your intended use is not permitted by statutory regulation or exceeds the permitted use, you will need to obtain permission directly from the copyright holder. To view a copy of this licence, visit http://creativecommons.org/licenses/by$\mathrm{nc} / 4.0 /$.

\section{REFERENCES}

1. Haneda $M$, Noda $M$, Origasa $H$, et al. Japanese clinical practice guideline for diabetes 2016. J Diabetes Investig. 2018;9:657-97.

2. Buse JB, Wexler DJ, Tsapas A, et al. 2019 Update to: Management of hyperglycemia in type 2 diabetes, 2018. A consensus report by the American Diabetes Association (ADA) and the European Association for the Study of Diabetes (EASD). Diabetes Care. 2018;2020:487-93.

3. Food and Drug Administration, USA. Ozempic ${ }^{\circledR}$ prescribing information. 2020.

4. Food and Drug Administration, USA. Trulicity ${ }^{\circledR}$ prescribing information. 2020.

5. Food and Drug Administration, USA. Victoza ${ }^{\circledR}$ prescribing information. 2020.

6. Marso SP, Bain SC, Consoli A, et al. Semaglutide and cardiovascular outcomes in patients with type 2 diabetes. N Engl J Med. 2016;375:1834-44.
7. Marso SP, Daniels GH, Brown-Frandsen $\mathrm{K}$, et al. Liraglutide and cardiovascular outcomes in type 2 diabetes. N Engl J Med. 2016;375:311-22.

8. Gerstein HC, Colhoun HM, Dagenais GR, et al. Dulaglutide and cardiovascular outcomes in type 2 diabetes (REWIND): a double-blind, randomised placebo-controlled trial. Lancet. 2019;394:121-30.

9. Buckley ST, Bækdal TA, Vegge A, et al. Transcellular stomach absorption of a derivatized glucagon-like peptide-1 receptor agonist. Sci Transl Med. 2018;10: eaar7047.

10. Harashima S, Nishimura A, Inagaki N. Attitudes of patients and physicians to insulin therapy in Japan: an analysis of the Global Attitude of Patients and Physicians in Insulin Therapy study. Expert Opin Pharmacother. 2017;18:5-11.

11. Sikirica MV, Martin AA, Wood R, et al. Reasons for discontinuation of GLP1 receptor agonists: data from a real-world cross-sectional survey of physicians and their patients with type 2 diabetes. Diabetes Metab Syndr Obes. 2017;10:403-12.

12. Billings LK, Handelsman Y, Heile M, Schneider D, Wyne K. Health-related quality of life assessments with once-weekly glucagon-like peptide-1 receptor agonists in type 2 diabetes mellitus. J Manag Care Spec Pharm. 2018;24(9-a Suppl):S30-41.

13. Abbasi J. Oral GLP-1 analog for type 2 diabetes on the horizon. JAMA. 2018;320:53.

14. Pharmaceutical and Medical Devices Agency, Japan. Rybelsus ${ }^{\circledR}$ prescribing information. 2020.

15. European Medicines Agency. Rybelsus ${ }^{\circledR}$ summary of product characteristics. 2020.

16. Food and Drug Administration, USA. Rybelsus ${ }^{\circledR}$ prescribing information. 2020.

17. Aroda VR, Rosenstock J, Terauchi Y, et al. PIONEER 1: randomized clinical trial comparing the efficacy and safety of oral semaglutide monotherapy with placebo in patients with type 2 diabetes. Diabetes Care. 2019;42:1724-32.

18. Rodbard HW, Rosenstock J, Canani LH, et al. Oral semaglutide versus empagliflozin in patients with type 2 diabetes uncontrolled on metformin: the PIONEER 2 trial. Diabetes Care. 2019;42:2272-81.

19. Rosenstock J, Allison D, Birkenfeld AL, et al. Effect of additional oral semaglutide vs sitagliptin on glycated hemoglobin in adults with type 2 diabetes uncontrolled with metformin alone or with sulfonylurea: the PIONEER 3 randomized clinical trial. JAMA. 2019;321:1466-80. 
20. Pratley R, Amod A, Hoff ST, et al. Oral semaglutide versus subcutaneous liraglutide and placebo in type 2 diabetes (PIONEER 4): a randomised, doubleblind, phase 3a trial. Lancet. 2019;394:39-50.

21. Mosenzon O, Blicher TM, Rosenlund S, et al. Efficacy and safety of oral semaglutide in patients with type 2 diabetes and moderate renal impairment (PIONEER 5): a placebo-controlled, randomised, phase 3a trial. Lancet Diabetes Endocrinol. 2019;7: 515-27.

22. Husain M, Birkenfeld AL, Donsmark M, et al. Oral semaglutide and cardiovascular outcomes in patients with type 2 diabetes. $\mathrm{N}$ Engl J Med. 2019;381:841-51.

23. Pieber TR, Bode B, Mertens A, et al. Efficacy and safety of oral semaglutide with flexible dose adjustment versus sitagliptin in type 2 diabetes (PIONEER 7): a multicenter, open-label, randomised, phase 3a trial. Lancet Diabetes Endocrinol. 2019;7:528-39.

24. Zinman B, Aroda VR, Buse JB, et al. Efficacy, safety, and tolerability of oral semaglutide versus placebo added to insulin with or without metformin in patients with type 2 diabetes: the PIONEER 8 trial. Diabetes Care. 2019;42:2262-71.

25. Yamada Y, Katagiri H, Hamamoto Y, et al. Doseresponse, efficacy, and safety of oral semaglutide monotherapy in Japanese patients with type 2 diabetes (PIONEER 9): a 52-week, phase 2/3a, randomised, controlled trial. Lancet Diabetes Endocrinol. 2020;8:377-91.

26. Yabe D, Nakamura J, Kaneto H, et al. Safety and efficacy of oral semaglutide versus dulaglutide in Japanese patients with type 2 diabetes (PIONEER 10): an open-label, randomised, activecontrolled, phase $3 \mathrm{a}$ trial. Lancet Diabetes Endocrinol. 2020;8:392-406.

27. Ma RCW, Chan JCN. Type 2 diabetes in East Asians: similarities and differences with populations in Europe and the United States. Ann N Y Acad Sci. 2013;1281:64-91.

28. Ishii H. Development and psychometric validation of the Diabetes Therapy-Related QOL (DTR-QOL) questionnaire. J Med Econ. 2012;15:556-63.

29. Aroda VR, Saugstrup T, Buse JB, Donsmark M, Zacho J, Davies MJ. Incorporating and interpreting regulatory guidance on estimands in diabetes clinical trials: the PIONEER 1 randomized clinical trial as an example. Diabetes Obes Metab. 2019;21: 2203-10.

30. Little RJA, Rubin DB. Statistical analysis with missing data. New York, NY. Wiley; 1987.
31. Igarashi A, Bekker Hansen B, Langer J, et al. Preference for oral and injectable GLP-1 RA therapy profiles in Japanese patients with type 2 diabetes: a discrete choice experiment. Adv Ther. 2020. https://doi.org/10.1007/s12325-020-01561-1.

32. Ishii $H$, Shin $H$, Tosaki $T$, et al. Reproducibility and validity of a questionnaire measuring treatment burden on patients with type 2 diabetes: Diabetic Treatment Burden Questionnaire (DTBQ). Diabetes Ther. 2018;9:1001-19.

33. Takase T, Nakamura A, Yamamoto C, et al. Improvement in treatment satisfaction after switching from liraglutide to dulaglutide in patients with type 2 diabetes: a randomized controlled trial. J Diabetes Investig. 2019;10:699-705.

34. Ishii $\mathrm{H}$, Niiya $\mathrm{T}$, Ono $\mathrm{Y}$, et al. Improvement of quality of life through glycemic control by liraglutide, a GLP-1 analog, in insulin-naive patients with type 2 diabetes mellitus: the PAGE1 study. Diabetol Metab Syndr. 2017;9:3.

35. Ishii $\mathrm{H}$, Onishi $\mathrm{Y}$, Oura $\mathrm{T}$, et al. Once-weekly dulaglutide with insulin therapy for type 2 diabetes: efficacy and safety results from a phase 4 , randomized, placebo-controlled study. Diabetes Ther. 2020;11:133-45.

36. Ishii H, Suzaki Y, Miyata Y, Matsui S. Randomized multicenter evaluation of quality of life and treatment satisfaction in type 2 diabetes patients receiving once-weekly trelagliptin versus a daily dipeptidyl peptidase-4 inhibitor. Diabetes Ther. 2019;10:1369-80.

37. Mita T, Katakami N, Shiraiwa T, et al. The influence of sitagliptin on treatment-related quality of life in patients with type 2 diabetes mellitus receiving insulin treatment: a prespecified sub-analysis. Diabetes Ther. 2017;8:693-704.

38. Mita T, Yoshii H, Chimori $\mathrm{H}$, et al. The effect of linagliptin versus metformin treatment-related quality of life in patients with type 2 diabetes mellitus. Diabetes Ther. 2019;10:119-34.

39. Kato M, Sakai K, Saito K, Tsutsui K, Yamashita S, Kato N. Efficacy and safety of ipragliflozin in Japanese patients with type 2 diabetes receiving conventional therapy: clinical implication of the importance of exercise habits during treatment with ipragliflozin. Diabetol Int. 2017;8:275-85.

40. Ishii H, Takamura H, Nishioka Y, et al. Quality of life and utility values for cost-effectiveness modeling in Japanese patients with type 2 diabetes. Diabetes Ther. 2020;11:2931-43. 